\title{
Social-Cognitive Predictors of Health Behavior: Action Self-Efficacy and Coping Self-Efficacy
}

\author{
Ralf Schwarzer \\ Freie Universität Berlin
}

\author{
Britta Renner \\ Ernst-Moritz-Arndt-Universität Greifswald
}

\begin{abstract}
The effects of social-cognitive variables on preventive nutrition and behavioral intentions were studied in $\mathbf{5 8 0}$ adults at 2 points in time. The authors hypothesized that optimistic self-beliefs operate in 2 phases and made a distinction between action self-efficacy (preintention) and coping self-efficacy (postintention). Risk perceptions, outcome expectancies, and action self-efficacy were specified as predictors of the intention at Wave 1. Bebavioral intention and coping self-efficacy served as mediators linking the 3 predictors with low-fat and high-fiber dietary intake 6 months later at Wave 2. Covariance structure analysis yielded a good model fit for the total sample and 6 subsamples created by a median split of 3 moderators: gender, age, and body weight. Parameter estimates differed between samples; the importance of perceived self-efficacy increased with age and weight.
\end{abstract}

Key words: health cognitions, risk appraisals, self-efficacy, outcome expectancies, preventive nutrition, body weight

The Berlin Risk Appraisal and Health Motivation Study (BRAHMS) was designed to examine the social-cognitive determinants of health behaviors, such as physical exercise, smoking, alcohol consumption, and preventive nutrition. In the study reported in this article, our focus is on self-reported nutrition. Eating a healthy diet low in saturated fat and high in fiber is a common medical recommendation. According to current medical knowledge, such nutrition helps reduce the risk of cardiovascular disease and other ailments. However, most people do not adhere to this advice, and many have not even developed an explicit intention to adopt it.

Three factors specified by social-cognitive health behavior theories were considered as possible predictors: (a) risk appraisals, defined as one's perceived vulnerability compared to that of others; (b) behavior-specific outcome expectancies (i.e., expected benefits of preventive nutrition); and (c) self-efficacy beliefs in the face of obstacles and barriers to adopt health behaviors. Moreover, the roles of gender, age, and body weight were examined.

Ralf Schwarzer, Gesundeheitspsychologie, Freie Universität Berlin, Berlin, Germany; Britta Renner, Department of Psychology, Ernst-MoritzArndt-Universităt Greifswald, Griefswald, Germany.

This research was supported by the Deutsche Forschungsgemeinschaft and the Techniker Krankenkasse fir Berlin und Brandenburg. We thank Andre Hahn and Thomas von Lengerke for their collaboration on this project and Bärbel Knăuper, Gerdamarie Schmitz, and Lars Satow for their helpful comments on the first draft.

Correspondence concerning this article should be addressed to Ralf Schwarzer, Gesundheitspsychologie, Freie Universitat Berlin, Habelschwerdter Allee 45, 14195 Berlin, Germany. Electronic mail may be sent to health@zedat.fu-berlin.de.

\section{Perceived Self-Efficacy and Preventive Nutrition}

The construct of self-efficacy represents one core aspect of social-cognitive theory (Bandura, 1997). Whereas outcome expectancies refer to the perception of the possible consequences of one's action, perceived self-efficacy refers to personal action control or agency. A person who believes that he or she can produce a desired effect can conduct a more active and self-determined life course.

Dieting, weight control, and preventive nutrition can be governed by self-efficacy beliefs within such a self-regulatory cycle. It has been found that self-efficacy operates best in concert with general changes in lifestyle, including physical exercise and provision of social support. Self-confident clients of intervention programs were less likely than other clients to relapse into their previous unhealthy diet (Bagozzi \& Edwards, 1998; Brug, Hospers, \& Kok, 1997; Fuhrmann \& Kuhl, 1998; Gollwitzer \& Oettingen, 1998). Chambliss and Murray (1979) found that people who were overweight were most responsive to behavioral treatment when they had a high sense of self-efficacy. Body weight, age, and gender should be considered when the motivation to change one's nutrition is studied. Setting preventive nutrition goals and making self-regulatory attempts require optimistic self-beliefs, an assumption that has been discussed in social cognition models of health behavior.

\section{Social Cognition Models and Health Behavior}

Health behavior theories describe and explain how and why individuals refrain from risk behaviors and adopt health behaviors (Conner \& Norman, 1996; Wallston, 1994; Weinstein, 1993). According to Abraham, Sheeran, and Johnston (1998), the common view is emerging that several ingredients of such models are necessary, among them (a) intentions, (b) perceived self-efficacy, 
and (c) outcome expectancies. One such theory is the health action process approach (HAPA; Schwarzer, 1992, 1999; Schwarzer \& Fuchs, 1996). HAPA was built around these characteristics from a dynamic self-regulation perspective. This approach suggests a distinction between preintentional motivation processes and postintentional volition processes.

In the initial motivation phase, a person develops an intention (or goal) to act. This is mainly inspired by three kinds of cognitions: risk perception, outcome expectancies, and perceived selfefficacy. After a goal has been set within the motivation phase, individuals enter the volition phase, in which they plan the details, try to act, invest effort, persist, possibly fail, and finally recover. Thus, there are at least two distinct phases, one that leads to a behavioral intention and another that leads to actual behavior.

According to this theory, the intention to adopt a valued health behavior (such as preventive nutrition) depends mainly on three sets of cognitions: (a) the belief that one is at risk for disease ("I have a high risk of getting a heart attack because of my high cholesterol level and body weight"), (b) the belief that behavioral change would reduce a health threat ("If I eat healthful foods, I will reduce my cardiovascular risk"), and (c) the belief that one is sufficiently capable of exercising control over a difficult behavior ("I am capable of controlling my healthful diet in spite of sweet temptations"). Risk perceptions serve predominantly to set the stage for a contemplation process early in the motivation phase, but they do not extend beyond this phase. Similarly, outcome expectancies are chiefly seen as being important in the motivation phase, when a person balances the pros and cons of certain behavior consequences, but they may lose their predictive power after a personal decision has been made. However, if one does not believe in one's capability to perform a desired action (i.e., lack of perceived self-efficacy), one will be unable to initiate and maintain it. Beliefs can be the moving force while a person proceeds through a self-regulatory cycle. At different points in time, different patterns of social-cognitive predictor sets may emerge.

\section{Phase-Specific Self-Efficacy}

Optimistic self-beliefs may be phase-specific within a selfregulatory cycle. For example, some individuals may have high confidence in their ability to set ambitious goals and to take initiative but little confidence in their ability to maintain the desired behaviors. In contrast, others may have high confidence in their ability to resist temptation and to recover from setbacks but little confidence in getting started. Thus, perceived self-efficacy is seen as functional at different levels and at different points in time within a self-regulatory goal attainment process. It might be useful to subdivide the construct in a phase-specific manner in order to characterize these functions (see Marlatt, Baer, \& Quigley, 1995). Action self-efficacy makes a difference in the preactional phase. Individuals high in self-efficacy imagine success scenarios, anticipate potential outcomes of diverse strategies, and take the initiative to try to adopt a new behavior (Bagozzi \& Edwards, 1998). Those with less self-efficacy, on the other hand, imagine failure scenarios, harbor self-doubts, and procrastinate. Coping selfefficacy, on the other hand, describes optimistic beliefs about one's capability to deal with barriers that arise during the maintenance period. A new health behavior might turn out to be much more difficult to adhere to than expected, but a self-efficacious person responds confidently with better strategies, more effort, and prolonged persistence to overcome such hurdles. Once an action has been taken, individuals with high coping self-efficacy invest more effort and persist longer than those who are not self-efficacious. When setbacks occur, they recover more quickly and maintain commitment to their goals.

The study we report here did not allow a test of this entire model, but it did include crucial variables. Therefore, it permitted the exploration of some of the proposed relationships and helped to establish empirically the conceptual distinction between action self-efficacy and coping self-efficacy. The research design covered the three predictors within the motivation phase (risk perception, outcome expectancies, and action self-efficacy) and the intention to adopt a healthy diet. The study also included coping selfefficacy in the volition phase half a year later, covering two preventive nutrition behaviors. We assumed that the intention as well as the behaviors could be predicted by social-cognitive variables, which would corroborate previous research findings. The questions were, in particular, how strong the associations would be and what kind of prediction pattern would emerge. Additional questions were whether the hypothesized general causal model could be replicated within subsamples and whether the relationships were moderated by variables such as age, body weight, and gender. We assumed that preventive nutrition is more salient in older and overweight persons, which might affect the causal structure or the strength of the empirical associations.

\section{Method}

\section{Participants}

Participants were 580 residents of Berlin who came to four different locations (two universities and two city halls). Informed consent was obtained. The average age was 43 years, and $48 \%$ were male. Five hundred twenty-six respondents $(91 \%)$ from the first wave completed the follow-up questionnaire 6 months later. After listwise deletion of missing data on all variables, a longitudinal sample of 524 persons remained. Dropout analysis showed that the average age of the longitudinal sample was 7 years younger than that of the dropout sample, $t(578)=3.13, p=.002$. However, there was no significant difference between the two groups in terms of body weight, $t(578)=.47, p=.64$, and gender, $\chi^{2}(1, N=578)=$ $.89, p=.35$.

\section{Procedure}

Participants were recruited through advertisements placed in local newspapers in Berlin. Furthermore, a letter describing the study was sent to clients of the Technician's Health Insurance company who lived near the study locations. On arriving at the study site, participants were told that the study was part of a community-wide effort to collect health information from the population at various locations across Berlin. They completed a questionnaire that included 22 items measuring health-related cognitions and behaviors. Afterward, they were thanked and received an invitation to take part in the second wave. Six months later, participants were assessed again in the same manner.

\section{Measures}

A set of 22 questionnaire items related to preventive nutrition was designed to assess seven latent variables, four at Time 1 and three at Time 2. Risk perception, outcome expectancies, action self-efficacy, and 
intentions were measured first; coping self-efficacy, low-fat dietary intake, and high-fiber dietary intake were measured half a year later.

All items are translated from German. Cronbach's alphas are cited to give an impression of the internal consistencies, although multipleindicator models were used instead of sum scores of the scales.

For the assessment of risk perception, three items indicating relative vulnerability were used (Cronbach's $\alpha=.78$ ): "If I compare myself with others of my age and gender, I estimate the likelihood of experiencing (a) heart disease..., (b) high blood pressure..., (c) a stroke..." Responses were given on 7-point scales anchored at much below average $(-3)$ and much above average $(+3)$.

Outcome expectancies were measured by three items (Cronbach's $\alpha=$ .81). Participants were asked, "What do you think will be the personal consequences for yourself if you adopt a low-fat, high-fiber diet?" After this header, responses were elicited to three specific questions: "If I stick to a low-fat, high-fiber diet, then ... (a) I would feel physically more attractive, (b) I would feel better mentally, and (c) I would have no (or fewer) body weight problems." Responses were made on 7-point scales.

Perceived self-efficacy was measured in two ways: as an action selfefficacy at Time 1 and half a year later as coping self-efficacy at Time 2 The general stem for all items was, "How certain are you that you could overcome the following barriers?" The first measure, action self-efficacy, consisted of two indicators $(r=.79)$ : "I can manage to stick to healthful food, (a) ... even if I have to make a detailed plan and (b) ... even if I have to rethink my entire way of nutrition." These items were designed to assess perceived action self-efficacy in the preactional phase, when individuals set their goals or anticipate difficulties in the planning phase.

The second measure, coping self-efficacy, consisted of three indicators (Cronbach's $\alpha=.85$ ): "I can manage to stick to healthful food, (a) . . . even if I have to try several times until it works, (b) ... even if I need a long time to develop the necessary routines, and (c) ... even if I do not receive a great deal of support from others when making my first attempts." These iterns were designed to assess perceived coping self-efficacy in the postintentional phase, when a person is directly confronted with barriers and setbacks and has to overcome them or is concerned about the appropriate initiative. Responses were made on 4-point scales, ranging from not at all true (1) to exactly true (4).

The intention to adopt preventive nutrition habits was assessed with four items (Cronbach's $\alpha=.91$ ): "I intend to eat only a very small amount of fat (such as saturated fat, cheese, butter) over the next months," "I intend to live a healthier life," "I intend to eat healthful foods over the next months," and "I intend to invest more into my health." Responses were made on 4-point scales.

There were two dimensions of self-reported nutrition behavior half a year later, one related to a high-fiber dietary intake, with three items (Cronbach's $\alpha=.81$ ): "I eat a lot of fresh fruit and vegetables," "I follow a high vitamin diet," and "I stick to a balanced diet." The other dimension, related to a low-fat dietary intake, contained four items (Cronbach's $\alpha=$ .82): "I follow a low-fat diet," "When I eat milk products or drink milk, 1 choose low-fat products (such as low-fat milk or yogurt)," "I avoid foods with cholesterol," and "I am aware of my calorie intake." Responses were made on 4-point scales. Some evidence for validity of these measures is given by correlations with self-reported food intake frequencies, based on a weekly estimate of specific foods. For example, the high-fiber diet sum score has a correlation of .27 with the frequency of consuming fruits and .34 with the frequency of eating green salads. The low-fat diet sum score has a correlation of .20 with the frequency of consuming eggs and .25 with the frequency of eating sausages (all $p \mathrm{~s}<.01$ ). These correlations are in line with those of Armitage and Conner (1999). However, self-reported food intake frequencies are of limited value as a measure of preventive nutrition because the amount of food actually consumed is influenced by body size, gender, age, and other factors, and the reported amount may not be recalled accurately over long periods of time.
Body weight and height were measured in order to calculate the individual body mass index (BMI; body weight [in $\mathrm{kg}] / \mathrm{m}^{2}$ ). Average BMI for women was $24(S D=3.8)$ and 26 for men $(S D=3.3$ ). Using criteria established by Bray (1978), we classified women with a BMI over 24 and men with a BMI over 25 as being overweight.

\section{Results}

\section{Covariance Structure Analysis}

To examine the associations between the variables, we chose a structural equations approach. Seven latent variables were specified, four at Time 1 and three at Time 2 . Risk perception as a latent variable included three indicators. The latent variable outcome expectancy was based on three indicators. Perceived action selfefficacy had two indicators. Intention was measured by four indicators. Perceived coping self-efficacy was represented by three indicators, measured half a year later. Low-fat diet was based on four behavioral indicators, and high-fiber diet was based on three behavioral indicators.

The first three latent variables were specified as predictors of the intention. The intention itself, along with coping self-efficacy, was specified as a predictor of the two behavioral dimensions.

This 22-indicator, 7-factor measurement model was applied not only to the entire data set, but also to various subsamples of young, old, slender, and overweight participants. Table 1 displays the correlation matrix.

All analyses were computed with the LISREL 8.12a program (Jöreskog \& Sörbom, 1993) based on correlation matrices of the total sample and various subsamples. Judgments about model fit were made jointly on the basis of the goodness-of-fit (GFI) index, the root-mean-square (RMR) residual, and the root-mean-square error of approximation (RMSEA). The estimated parameters of a model are a function of discrepancies between the observed covariance matrix and the reproduced one. Ideally, GFI should be above .90 , and RMR below .05. The chi-square value and its corresponding $p$ value are reported, but they did not serve as relevant criteria because they are only important when the assumption of multinormality is met, when sample sizes are not too large, or when differences between competing models or nested models are to be examined. Parameters were estimated with the unweighted least square method.

\section{Overall Analysis}

An initial overall analysis was performed with the total sample of 524 persons. The model fit was good, with GFI $=.98, \mathrm{RMR}=$ .051 , RMSEA $=.038(p=.73)$, and $\chi^{2}(197)=348.93, p<.001$. Table 2 displays the parameter estimates (standardized solution).

In the prediction of the intention, the hypothesized rank order (Schwarzer \& Fuchs, 1996) was confirmed: (a) outcome expectancies (.50), (b) action self-efficacy (.28), and (c) risk perception (.15). The unbiased estimated relationship between action selfefficacy (Time 1) and coping self-efficacy (Time 2) was .62. The latter, in conjunction with the intention, predicted the two behavior dimensions very well. Of the low-fat diet variation, $48 \%$ was accounted for, whereas of the high-fiber diet variation, $33 \%$ was accounted for jointly by intention and coping self-efficacy. Lowfat diet was better predicted by the intention, whereas high-fiber diet was better predicted by coping self-efficacy. 
Table 1

Correlation Matrix of 22 Indicators

\begin{tabular}{|c|c|c|c|c|c|c|c|c|c|c|c|c|c|c|c|c|c|c|c|c|c|c|}
\hline Indicator & 1 & 2 & 3 & 4 & 5 & 6 & 7 & 8 & 9 & 10 & 11 & 12 & 13 & 14 & 15 & 16 & 17 & 18 & 19 & 20 & 21 & 22 \\
\hline 1. Fiber 1 & - & & & & & & & & & & & & & & & & & & & & & \\
\hline 2. Fiber 2 & .519 & - & & & & & & & & & & & & & & & & & & & & \\
\hline 3. Fiber 3 & .622 & .621 & - & & & & & & & & & & & & & & & & & & & \\
\hline 4. Fat 1 & .352 & .385 & .409 & - & & & & & & & & & & & & & & & & & & \\
\hline 5. Fat 2 & .247 & .318 & .284 & .513 & - & & & & & & & & & & & & & & & & & \\
\hline 6. Fat 3 & .277 & .243 & .242 & .528 & .510 & - & & & & & & & & & & & & & & & & \\
\hline 7. Fat 4 & .342 & .340 & .345 & .580 & .590 & .557 & - & & & & & & & & & & & & & & & \\
\hline 8. Intent 1 & .279 & .238 & .286 & .471 & .516 & .483 & .531 & - & & & & & & & & & & & & & & \\
\hline 9. Intent 2 & .256 & .231 & .291 & .383 & .424 & .387 & .428 & .807 & - & & & & & & & & & & & & & \\
\hline 10. Intent 3 & .198 & .149 & .233 & .272 & .295 & .256 & .283 & .572 & .854 & - & & & & & & & & & & & & \\
\hline 11. Intent 4 & .211 & .228 & .275 & .309 & .329 & .322 & .351 & .632 & .898 & .670 & - & & & & & & & & & & & \\
\hline 12. Coping 1 & .187 & .352 & .290 & .281 & .284 & .273 & .307 & .279 & .257 & .195 & .234 & - & & & & & & & & & & \\
\hline 13. Coping 2 & .226 & .401 & .301 & .287 & .278 & .214 & .258 & .208 & .189 & .156 & .183 & .692 & - & & & & & & & & & \\
\hline 14. Coping 3 & .243 & .351 & .298 & .211 & .182 & .110 & .197 & .315 & .318 & .236 & .301 & .404 & .407 & - & & & & & & & & \\
\hline 15. Action 1 & .085 & .290 & .229 & .192 & .261 & .232 & .230 & .213 & .185 & .125 & .186 & .700 & .582 & .335 & - & & & & & & & \\
\hline 16. Action 2 & .208 & .315 & .259 & .187 & .154 & .103 & .186 & .260 & .293 & .215 & .304 & .394 & .362 & .788 & .365 & - & & & & & & \\
\hline 17. Outcome 1 & .213 & .192 & .241 & .338 & .278 & .298 & .316 & .378 & .432 & .375 & .376 & .157 & .164 & .248 & .149 & .177 & - & & & & & \\
\hline 18. Outcome 2 & .211 & .130 & .175 & .324 & .203 & .234 & .246 & .345 & .364 & .293 & .316 & .096 & .082 & .139 & .062 & .096 & .594 & - & & & & \\
\hline 19. Outcome 3 & .165 & .100 & .144 & .319 & .239 & .231 & .236 & .297 & .316 & .244 & .269 & .059 & .061 & .140 & .020 & .064 & .514 & .635 & - & & & \\
\hline 20. Risk 1 & -.038 & -.054 & -.135 & .083 & .088 & .075 & .009 & .084 & .119 & .124 & .086 & .021 & .003 & -.030 & .031 & .014 & .029 & .054 & .105 & - & & \\
\hline 21. Risk 2 & .004 & -.051 & -.072 & .109 & .151 & .096 & -.010 & .090 & .131 & .161 & .070 & -.028 & -.012 & -.043 & -.056 & -.037 & .013 & .026 & .088 & .530 & - & \\
\hline 22. Risk 3 & .045 & -.006 & -.057 & .113 & .145 & .092 & .0090 & .091 & .128 & .190 & .064 & .061 & .047 & -.013 & -.006 & -.003 & -.033 & -.010 & .067 & .467 & .653 & - \\
\hline
\end{tabular}


Table 2

LISREL Parameter Estimates for Total Sample $(N=524)$

\begin{tabular}{|c|c|c|c|c|c|c|c|}
\hline & Intention & $\begin{array}{c}\text { Low } \\
\text { fat }\end{array}$ & $\begin{array}{l}\text { High } \\
\text { fiber }\end{array}$ & $\begin{array}{c}\text { Coping } \\
\text { SE }\end{array}$ & $\begin{array}{c}\text { Action } \\
\text { SE }\end{array}$ & $\begin{array}{l}\text { Outcome } \\
\text { E }\end{array}$ & Risk \\
\hline \multicolumn{8}{|c|}{ Lambda-y Matrix } \\
\hline Fiberl & 一 & - & .77 & - & & & \\
\hline Fiber2 & - & - & .87 & - & & & \\
\hline Fiber3 & - & - & .88 & - & & & \\
\hline Fatl & - & .82 & - & - & & & \\
\hline Fat2 & - & .77 & - & - & & & \\
\hline Fat3 & - & .73 & - & - & & & \\
\hline Fat4 & - & .84 & - & - & & & \\
\hline Intent I & .91 & - & - & - & & & \\
\hline Intent2 & .93 & - & - & - & & & \\
\hline Intent3 & .75 & - & - & - & & & \\
\hline Intent 4 & .83 & - & - & - & & & \\
\hline Coping 1 & - & - & 一 & .94 & & & \\
\hline Coping 2 & - & - & - & .87 & & & \\
\hline Coping 3 & - & - & - & .76 & & & \\
\hline Action 1 & & & & & .81 & & \\
\hline Action2 & & & & & .90 & & \\
\hline Outcome1 & & & & & & .90 & \\
\hline Outcome2 & & & & & & .81 & \\
\hline Outcome3 & & & & & & .72 & \\
\hline Riskl & & & & & & & .64 \\
\hline Risk2 & & & & & & & .88 \\
\hline \multirow[t]{2}{*}{ Risk3 } & & & & & & & .79 \\
\hline & \multicolumn{4}{|c|}{ Beta matrix } & \multicolumn{3}{|c|}{ Gamma matrix } \\
\hline Intention & - & - & - & - & .29 & .51 & .15 \\
\hline Low fat & .59 & - & - & .24 & - & . & - \\
\hline High fiber & .31 & - & - & .40 & - & - & - \\
\hline Coping SE & - & - & - & - & .65 & - & - \\
\hline
\end{tabular}

Psi Matrix

\begin{tabular}{lllll} 
Intention & .54 & .52 & \\
Low fat & - & .22 & .67 & .58 \\
High fiber & - & - & - &. \\
Coping SE & - & & - \\
\hline
\end{tabular}

Note. $\mathrm{SE}=$ self-efficacy; $\mathrm{E}=$ expectancy.

Subsequent analyses focused on subgroups of participants. First, the data set was subdivided by gender, resulting in 253 men and 271 women. Both data matrices fitted equally well, which is in favor of the hypothesized general causal structure. Parameter estimates are not reported here because they were too similar in both subsamples and did not add information to the more relevant findings reported below.

Second, the entire sample was subdivided in terms of body weight. Women with a BMI above 24 and men with a BMI above 25 were classified as being overweight. There were 292 persons with a BMI below these criteria (for convenience labeled slim sample) and 232 with a BMI above (labeled heavy sample).

The slim sample. The fit for the persons who were slim was good, with GFI $=.97, \mathrm{RMR}=.06, \mathrm{RMSEA}=.045(p=.83)$, and $\chi^{2}(197)=310.93, p<.001$. Figure 1 displays the parameter estimates (standardized solution).

At Wave $1,40 \%$ of the variation of the behavioral intention was jointly accounted for by outcome efficacy (.48), action selfefficacy (.25), and risk perception (.19). At Wave 2, 45\% of the low-fat diet variance was accounted for by intention (.58) and coping self-efficacy $(.24)$. The opposite prediction pattern emerged for high-fiber diet (33\%), where coping self-efficacy was stronger (.35) than intention (.27).

The heavy sample. In the sample of those persons who were overweight, the fit was good, with GFI $=.98, \mathrm{RMR}=.06$, RMSEA $=.02(p=1.00)$, and $\chi^{2}(197)=212.68, p<.22$. Figure 2 displays the parameter estimates (standardized solution).

At Wave $1,47 \%$ of the variation of the behavioral intention was jointly accounted for by outcome efficacy (.43) and action selfefficacy (.39), but not by risk perception (.04). At Wave 2, 47\% of the low-fat diet variance was accounted for by intention (.47) and coping self-efficacy (.35). Again, a somewhat different prediction pattern emerged for high-fiber diet $(46 \%)$, with coping selfefficacy (.42) being as important as intention (.39). As for the indirect effects, self-efficacy exerted the strongest influence, whereas the influence of risk perception was nil.

In comparing the two subsamples that differed in body weight, we found that those who were overweight may be governed by self-efficacious thoughts more when changing their health behavior, whereas in the group with lower body 


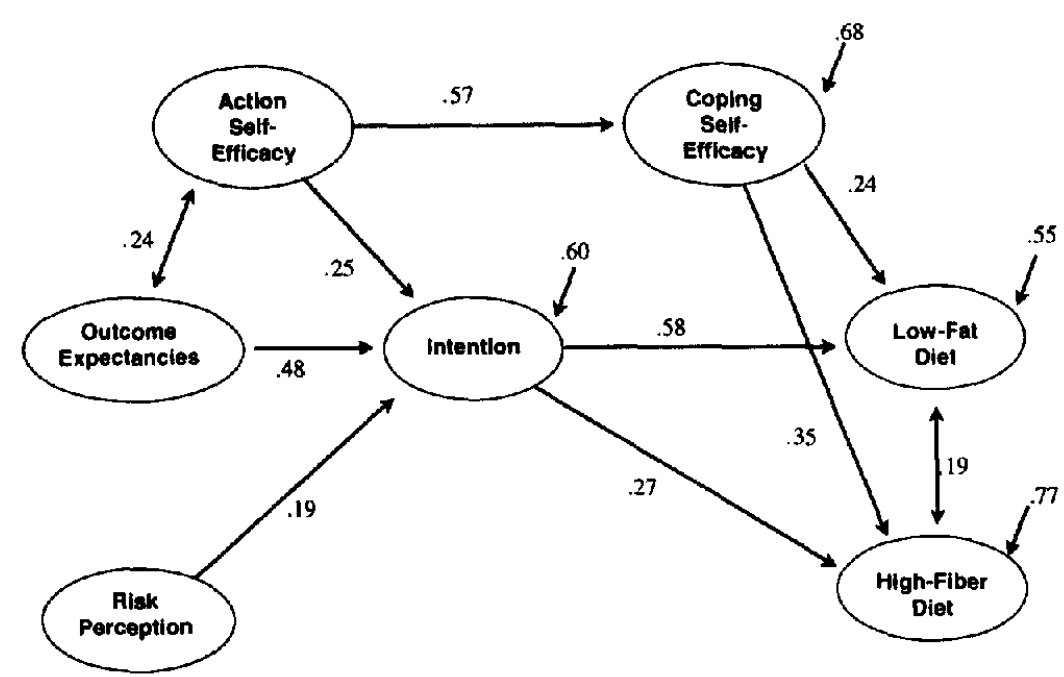

Figure 1. Health behavior change model in 292 persons with a below-average body mass index.

weight the outcome expectancy-intention chain appeared to be most conspicuous.

The young sample. Third, the entire sample was subdivided into two age groups. The young sample consisted of 248 persons ranging from 17 to 30 years of age (mean age $=25$ years). The fit was not so good, with GFI $=.95, \mathrm{RMR}=.078, \mathrm{RMSEA}=.06$ $(p=.02)$, and $\chi^{2}(197)=384.99, p<.001$. Figure 3 displays the parameter estimates (standardized solution).

At Wave 1,37\% of the variation of the behavioral intention was jointly accounted for by outcome efficacy (.56) and action selfefficacy (.13), but not by risk perception (.00). At Wave 2,39\% of the low-fat diet variance was accounted for by intention (.56) and coping self-efficacy (.21). Again, the opposite prediction pattern emerged for high-fiber diet (31\%), where coping self-efficacy was much stronger (.49) than intention (.20).

The old sample. The sample of older people consisted of 276 persons between 31 and 84 years of age (mean age $=50$ years).
The fit was good with GFI $=.98, \mathrm{RMR}=.059, \mathrm{RMSEA}=.03$ $(p=1.00)$, and $\chi^{2}(197)=245.63, p<.001$. Figure 4 displays the parameter estimates (standardized solution).

At Wave $1,55 \%$ of the variation of the behavioral intention was jointly accounted for by action self-efficacy (.45), outcome efficacy (.41), and risk perception (.22). At Wave $2,46 \%$ of the low-fat diet variance was accounted for by intention (.57) and coping self-efficacy $(.28)$. The same prediction pattern emerged for high-fiber diet $(37 \%)$, where coping self-efficacy was weaker (.31) than intention (.41). As for indirect effects, however, selfefficacy was the strongest predictor of behaviors within this sample, partly because of the high association between action and coping self-efficacy (.72).

In comparing the two subsamples of younger and older participants, we found that the general structure was well replicated in the latter, but not so well in the former. The patterns of influence also differed considerably.

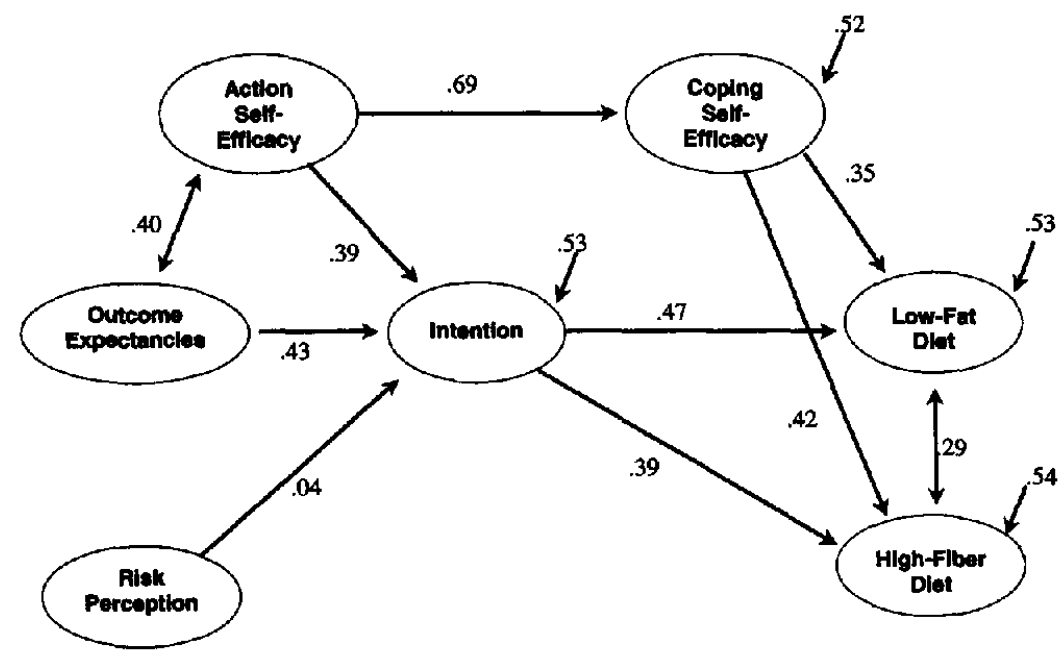

Figure 2. Health behavior change model in 232 persons with an above-average body mass index. 


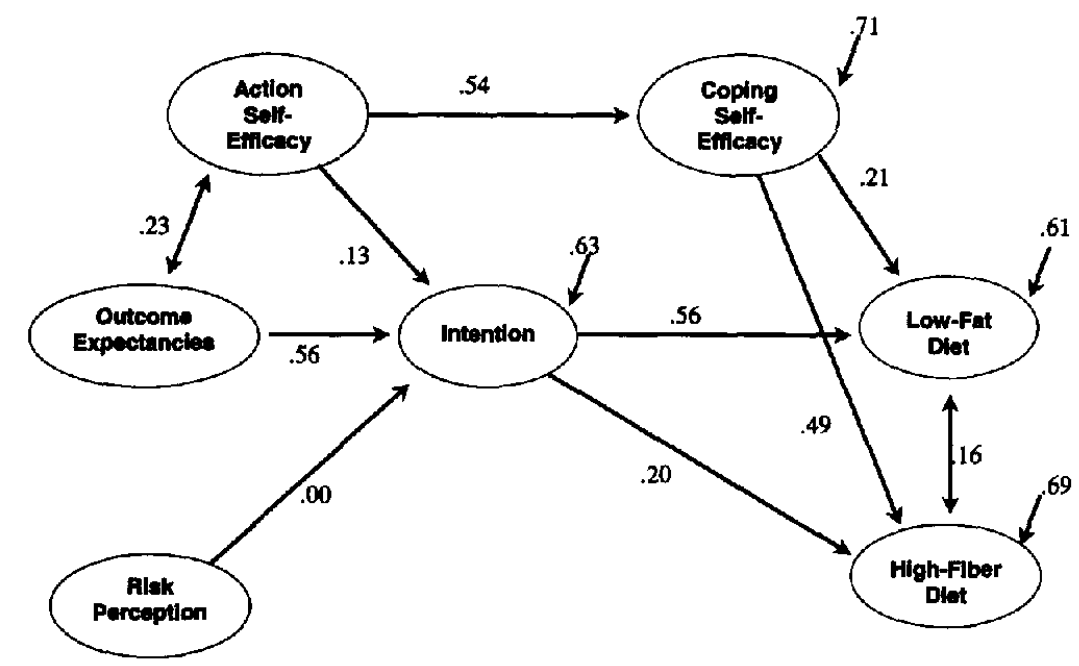

Figure 3. Health behavior change model in 248 persons below 31 years of age.

\section{Discussion}

As expected, the more self-efficacious individuals reported, on average, better nutrition behaviors. This finding can be understood as an interplay of several social-cognitive variables over time, as described in health behavior theories such as the HAPA (Schwarzer, 1992, 1999). The behavioral intention was well predicted by outcome expectancies and perceived self-efficacy, but less so by risk perception. The causal direction between the three predictors of intention remains speculative. Risk perception may set the stage for outcome expectancies, and the latter may trigger optimistic self-beliefs. However, there is no evidence in the present data for either this causal direction or for the opposite one. Experimental research is needed to shed more light on the causality issue. The predictive superiority of outcome expectancies over perceived self-efficacy in the motivation phase replicates earlier research (e.g., Dijkstra, De Vries, Roijackers, \& van Breukelen, 1998; Schwarzer \& Fuchs, 1996). It is possible that, depending on the particular context, one or the other construct becomes more central for the intention formation. The bivariate relationship between outcome expectancies and self-efficacy was moderate. Both constructs are regarded as necessary, but neither alone is sufficient. Individuals need to know the contingencies between behaviors and outcomes (e.g., outcome expectancies), but they also need to be confident that they really can perform the behavior in question (e.g., perceived self-efficacy).

All the leading health behavior theories suggest that the intention to change is probably the best predictor of subsequent behavior, unless unexpected barriers make the adoption of a health behavior unlikely. The present study has confirmed this assumption. Two measures of preventive nutrition behaviors were chosen, namely low-fat and high-fiber dietary intake, and both were well predicted by the behavioral intention that the research participants had expressed half a year before. Across all subsamples, the intention was more closely related to low-fat diet than to high-fiber

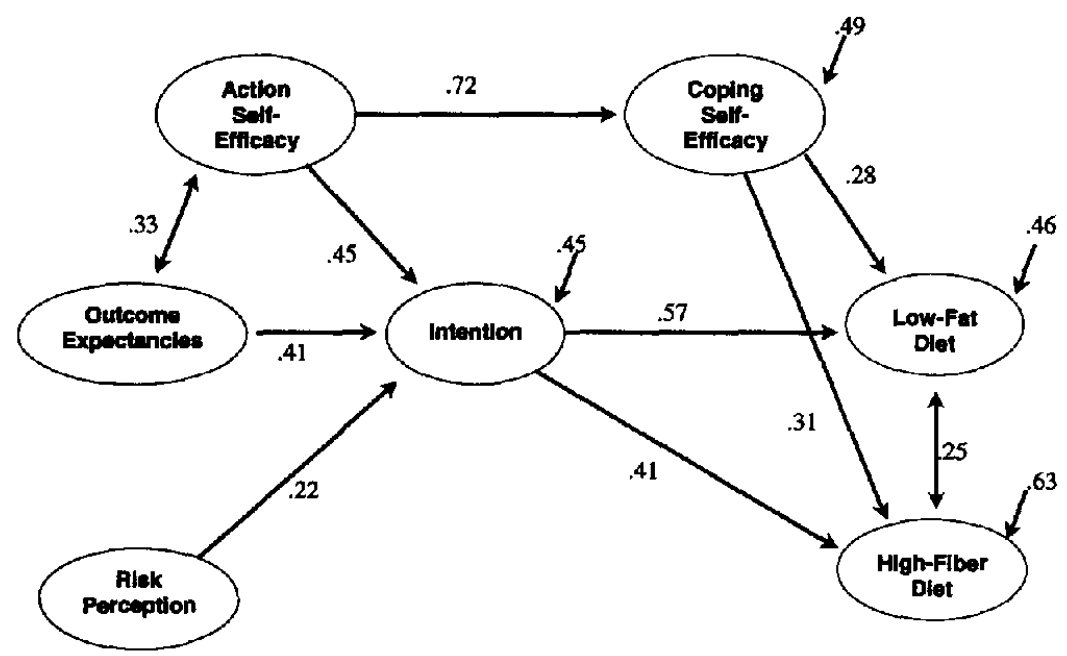

Figure 4. Health behavior change model in 276 persons above 30 years of age. 
diet. This is most probably due to the unbalanced measurement of the intention, which included four items, one of them explicitly referring to a low-fat diet, but none to a high-fiber diet.

A second assumption, based on the HAPA model, was the differential prediction within both the motivation phase and the volition phase. In the preintentional phase, the HAPA model assumes a weaker influence of perceived self-efficacy on intentions, compared to the influence by outcome expectancies. In the postintentional phase, however, it assumes a stronger influence of perceived self-efficacy on behavior compared to that by any other variable, except for intentions. Self-efficacious individuals are expected to develop more optimistic success scenarios for behavioral change, enhanced initiative, superior resistance in the face of temptation, and improved recovery after setbacks. Outcome expectancies, on the other hand, lose their importance at later stages of behavioral change, as confirmed by the present data.

The replication of the model fit across six subsamples, although not perfect for the sample of younger respondents, lends support to the assumption that the model chosen is meaningful and may serve as a useful heuristic for further research. Its main feature is the notion of phase-specific self-efficacy. Evidence emerged that selfbeliefs in the motivation phase can be distinguished from selfbeliefs during the maintenance phase. One may feel capable of making a decision and plan on it but less so of translating goals into action. Others might have difficulties to come up with an intention, but, after having done so, feel capable of taking action and maintaining the behavior.

Age and body weight turned out to be moderator variables, indicating a particular emphasis on action self-efficacy in older and overweight samples. This finding may emanate from increased task difficulty for these subgroups. Optimistic self-beliefs of being able to cope with adversity or barriers become more important when the task is personally significant and intricate.

The findings bear implications for health interventions. Previous interventions have focused on using risk communication to lower defensive optimism. The idea was to let people recognize how much they really are at risk for illness or injury. This traditional intervention strategy has not been very successful. The present theory would emphasize the opposite strategy by making people aware of their coping resources, that is, their capability to change a refractory behavior. Resource communication would thus be more effective than risk communication and would also be more acceptable. People should not be threatened by what they may lose, but rather should be challenged by what they could gain. Furthermore, interventions should be tailored to fit the needs of the recipients: For those who are in the preintentional phase, it would be most promising to improve outcome expectancies, whereas for their counterparts in the postintentional phase it would be more suitable to enhance coping self-efficacy (De Vries, Mudde, Dijkstra, \& Willemsen, 1998; Dijkstra et al., 1998; Prochaska, 1994; Sutton, 1996).

This study is limited, in particular, by its nonexperimental nature and by the self-reporting of nutrition behavior. A more refined measurement of the dependent variable is recommended (e.g., by a nutrition diary). Moreover, the dependent variable was only assessed at Time 2 , which did not allow the examination of behavioral change.

The cause-and-effect relationships found are based on theory and time lag. The fitted model, although well replicated, need not necessarily represent the only true model, and there may be others that also fit the data. Subdividing the total sample sequentially into three times two groups, according to gender, body weight, and age, may not be sufficient, and a factorial combination $(2 \times 2 \times 2=$ 8) would be superior; for example, it would allow for examining the group of older overweight women. In the present study, however, cell sizes would have become too small, and covariance structure analysis does not yield stable parameter estimates in small samples. Thus, more detailed analyses of this kind should be attempted in future research.

\section{References}

Abraham, C., Sheeran, P., \& Johnston, M. (1998). From health beliefs to self-regulation: Theoretical advances in the psychology of action control. Psychology and Health, 13, 569-591.

Armitage, C. J., \& Conner, M. (1999). The theory of planned behavior: Assessment of predictive validity and "perceived control." British Journal of Social Psychology, 38, 35-54.

Bagozzi, R. P., \& Edwards, E. A. (1998). Goal setting and goal pursuit in the regulation of body weight. Psychology and Health, 13, 593-621.

Bandura, A. (1997). Self-efficacy: The exercise of control. New York: Freeman.

Bray, G. (1978). Definition, measurement, and classification of the syndromes of obesity. International Journal of Obesity, 2, 99-112.

Brug, J., Hospers, H. J., \& Kok, G. (1997). Differences in psychosocial factors and fat consumption between stages of change for fat reduction. Psychology and Health, 12, 719-727.

Chambliss, C. A., \& Murray, E. J. (1979). Efficacy attribution, locus of control, and weight loss. Cognitive Therapy and Research, 3, 349-353.

Conner, M., \& Norman, P. (Eds.). (1996). Predicting health behaviour: Research and practice with social cognition models. Buckingham, England: Open University Press.

De Vries, H., Mudde, A. N., Dijkstra, A., \& Willemsen, M. C. (1998). Differential beliefs, perceived social influences, and self-efficacy expectations among smokers in various motivational phases. Preventive Medicine, 27(5/1), 681-689.

Dijkstra, A., De Vries, H., Roijackers, J., \& van Breukelen, G. (1998). Tailored interventions to communicate stage-matched information to smokers in different motivational stages. Journal of Consulting and Clinical Psychology, 66, 549-557.

Fuhmann, A., \& Kuhl, J. (1998). Maintaining a healthy diet: Effects of personality and self-reward versus self-punishment on commitment to and enactment of self-chosen and assigned goals. Psychology and Health, 13, 651-686.

Gollwitzer, P. M., \& Oettingen, G. (1998). The emergence and implementation of health goals. Psychology and Health, 13, 687-715.

Jöreskog, K. G., \& Sörbom, D. (1993). LISREL 8.03. Chicago: Scientific Software International.

Marlatt, G. A., Baer, J. S., \& Quigley, L. A. (1995). Self-efficacy and addictive behavior. In A. Bandura (Ed.), Self-efficacy in changing societies (pp. 289-315). New York: Cambridge University Press.

Prochaska, J. O. (1994). Strong and weak principles for processing from precontemplation to action on the basis of twelve problem behaviors. Health Psychology, 13(1), 47-51.

Schwarzer, R. (1992). Self-efficacy in the adoption and maintenance of health behaviors: Theoretical approaches and a new model. In $\mathbf{R}$. Schwarzer (Ed.), Self-efficacy: Thought control of action (pp. 217-242). Washington, DC: Hemisphere.

Schwarzer, R. (1999). Self-regulatory processes in the adoption and main- 
tenance of health behaviors: The role of optimism, goals, and threats. Joumal of Health Psychology, 4, 115-127.

Schwarzer, R., \& Fuchs, R. (1996). Self-efficacy and health behaviors. In M. Conner \& P. Norman (Eds.), Predicting health behavior: Research and practice with social cognition models (pp. 163-196). Buckingham, England: Open University Press.

Sutton, S. R. (1996). Can "stages of change" provide guidance in the treatment of addictions? A critical examination of Prochaska and
DiClemente's model. In G. Edwards \& C. Dare (Eds.), Psychotherapy, psychological treatments, and the addictions (pp. 189-205). Cambridge, England: Cambridge University Press.

Wallston, K. A. (1994). Theoretically based strategies for health behavior change. In M. P. O'Donnell \& J. S. Harris (Eds.), Health promotion in the workplace (2nd ed., pp. 185-203). Albany, NY: Delmar.

Weinstein, N. D. (1993). Testing four competing theories of healthprotective behavior. Health Psychology, 12, 324-333. 